\title{
Management of severe spasticity with intrathecal baclofen delivered by a manually operated pump
}

\author{
Victor Patterson, Michael Watt, Dermot Byrnes, Diana Crowe, Albert Lee
}

\begin{abstract}
Intrathecal baclofen abolishes spasticity in many patients with neurological diseases but there are few studies on its long-term effectiveness. Since 1986 a manually operated subcutaneous pump has been used to deliver baclofen intrathecally in 21 patients with a follow up of at least one year. Most patients had multiple sclerosis and all were wheelchair-bound. Sixteen patients had a complete and sustained benefit. In four other patients the treatment was effective in the short term but not in the long term. In the remaining patient the pump never worked. Complications included meningitis, pump failure, erosion through the skin, and baclofen overdose. Nevertheless, only three patients have asked to discontinue the treatment. We conclude that intrathecal baclofen, delivered by a manually operated implanted pump, is an effective treatment for severe spasticity in most patients.
\end{abstract}

$(\Im$ Neurol Neurosurg Psychiatry 1994;57:582-585)

Reports in $1984^{1}$ and $1985^{2}$ suggested that intrathecal baclofen is an effective treatment for severe spasticity. We were impressed with the dramatic effect on spasticity of an intrathecal bolus of baclofen and in 1986 began to use a manually operated implanted pump to administer the drug on a permanent basis. We report here our six years of experience with this technique.

Neurology, Roya

Victoria Hospital,

Belfast, UK

V Patterson

$M$ Watt

A Lee

Department of Neurosurgery, Royal

Victoria Hospital,

Belfast, UK

D Byrnes

Regional

Pharmaceutical

Laboratory, Belfast

City Hospital, Belfast

UK

D Crowe

Correspondence to:

Dr V H Patterson,

Department of Neurology,

Royal Victoria Hospital,

Grosvenor Road, Belfast

BT12 6BA, UK.

Received 6 April 1993

and in revised form

3 September 1993.

Accepted 23 September

1993 if they fulfilled the following criteria: (a) severe leg spasticity or spasms unresponsive to drugs by mouth at the maximum tolerated doses; $(b)$ leg spasticity or spasms which interfered with everyday life as judged either by the patient or their carer; $(c)$ a good response of the spasticity or spasms to an intrathecal bolus of baclofen; and $(d)$ informed consent of the patient and family.

\section{OUTCOME MEASURES}

Hypertonia, spasms, and the improvement in quality of life as judged by the patient and carers were used as the three main measures of outcome. Tone was measured using the Ashworth scale. ${ }^{3}$ This is an ordinal scale which grades tone in the muscle between 1 (normal) and 5 (where the limb is fixed). Care was taken to exclude fixed contractures due to muscle shortening. Hip flexion, extension, and abduction, and knee flexion and extension were tested in all patients. The three worst movements from each leg were summed to give the patient's spasticity score. Spasms were scored for each leg: 0 for absent, 1 for movement-induced, 2 for touch-induced, and 3 for spontaneous. The sum of these scores gave the patient's spasm score. We determined the Barthel index and asked patients and carers specific questions about sitting, bathing, toileting, and dressing as we found this more sensitive than complex quality of life scales in measuring ease of care-giving.

INTRATHECAL BACLOFEN INJECTION

Various strengths of baclofen injection ranging from 50 to $3000 \mu \mathrm{g} / \mathrm{ml}$ were prepared in batches by the central pharmaceutical production unit, Royal Victoria Hospital, using baclofen powder obtained from either CibaGeigy or Bufa BV. Where patients required different strengths from those available these were prepared aseptically in the pharmacy department, Royal Victoria Hospital by diluting a more concentrated injection. Most patients were started with an injection of $50 \mu \mathrm{g}$ given at lumbar puncture. Tone and spasms were assessed at three hours and, if the spasticity score was not reduced to between six and 12 or spasms were not completely abolished, the procedure was repeated at intervals of two days using increasing doses of baclofen up to a maximum dose of $300 \mu \mathrm{g}$. Most patients responded to $200 \mu \mathrm{g}$ or less.

PUMP

We used a Secor manually operated pump (Cordis Europa NV) which has previously been used for the administration of intrathecal morphine to patients with intractable pain. ${ }^{4}$ 
The pump delivers a bolus of $0 \cdot 1 \mathrm{ml}$ when two buttons are pressed in sequence. The drug cannot be delivered by pressure on the device or by any other button-pressing manoeuvre. The pump contains a reservoir which holds $12 \mathrm{ml}$ and which is filled percutaneously through a filling dome by a 25 -gauge needle. A valve between the dome and the reservoir prevents reflux occurring and is opened by pressure from the filling needle on a firm disc.

\section{IMPLANTATION TECHNIQUE}

The pump was implanted under general anaesthesia. A 14-gauge Tuohy needle was introduced into the intrathecal space at the second lumbar interspace via a small midline lumbar incision and the silastic catheter was passed through the needle so that its tip lay at approximately $\mathrm{T} 12$. The catheter was then tunnelled subcutaneously to the lower ribs anteriorly where a pocket was fashioned to accommodate the pump: this had to be sufficiently superficial to allow palpation of the dome and buttons. The pump was then primed, filled, and connected to the catheter. For the first four implantations the pump was used once daily for the first five days but for the rest of the series the pump was not used at all for the first 14 days, at which time the skin sutures were removed. Initially the pump was filled with baclofen at implantation but later we used $0.9 \%$ sodium chloride solution, changing this over to baclofen when the pump was first used.

\section{REFILIING PROCEDURE}

Pumps were refilled either when it was calculated that they would be empty (at intervals of two months for twice daily dosage) or to change the concentration of baclofen solution. The filling dome over the pump was cleaned for five minutes with iodine solution and the reservoir was then emptied using a short 25gauge needle. This procedure was carried out under sterile conditions with the operator wearing a mask, gloves, and gown. The volume removed was recorded and $12 \mathrm{ml}$ baclofen solution was injected through the same needle. At the first and second refill the patients were kept in hospital and the spasticity scores measured four times daily to check that there was an even effect throughout the day. Dosage was adjusted either by increasing the frequency of administration or by increasing the concentration of baclofen in the reservoir up to $3000 \mu \mathrm{g} / \mathrm{ml}$, a maximum limited by the solubility of baclofen.

\section{IN VITRO ASSESSMENT OF COMPATIBILITY}

Three Secor implantable drug pumps (supplied by Cordis Europa) were filled and primed with $12 \mathrm{ml}$ baclofen injection of strength $250 \mu \mathrm{g} / \mathrm{ml}$. They were then transferred to wide-necked glass jars containing sodium chloride solution $(0.9 \% \mathrm{w} / \mathrm{v})$ and these were incubated at $37^{\circ} \mathrm{C}$. At seven and 18 weeks a $2-\mathrm{ml}$ sample of the baclofen solution from each pump was withdrawn aseptically through the filling dome and analysed for the concentration of baclofen and its breakdown product. ${ }^{5}$ At 18 weeks the pumps were returned to their manufacturers for detailed examination.

\section{IN VIVO ASSESSMENT OF STABILITY}

Samples obtained from four consecutive refills in patients 1 and 2 were analysed for baclofen content.

\section{BACLOFEN ASSAY}

In the early stages of the study samples were diluted to $0.001 \% \mathrm{w} / \mathrm{v}$ with $0.1 \mathrm{~m}$ hydrochloric acid and were analysed by ultraviolet spectroscopy. The absorbances of the samples were measured at $220 \mathrm{~nm}$ and the percentage of baclofen in the samples calculated from the formula: \% baclofen $=100$ (absorbance sample/absorbance standard). The reproducibility of the method was determined by analysing 10 replicate dilutions of baclofen USPRS. At a concentration of $0.001 \% \mathrm{w} / \mathrm{v}$ the coefficient of variation was $1.55 \%$. On heating baclofen rearranges its structure to form a lactam and the content of this is limited in baclofen tablets by the British ${ }^{6}$ and United States pharmacopoeias, ${ }^{7}$ where it is measured by thin-layer chromatography (TLC) and high-performance liquid chromatography (HPLC) respectively. We used a slight modification of the TLC method in the British pharmacopoeia to detect concentrations of lactam equivalent to $1 \%$ breakdown of baclofen. Since 1990 the availability of a suitable instrument has enabled the development of a stability indicating validated HPLC method based on that described by Harrison. ${ }^{8}$ This has become the chosen method for the determination of the baclofen and lactam content of samples of the pump solution and for the quality control testing of batches of baclofen injection.

\section{Results}

The table summarises the overall results.

\section{EFFECT ON SPASTICITY AND SPASMS}

Of the 21 patients who had pumps inserted 16 had a complete and sustained benefit, with spasticity reduced to normal or near normal (spasticity score between 6 and 12) and complete relief of spasms throughout the day. In four patients the treatment was effective in the short term but not in the long term; in the remaining patient the pump was removed without ever having been used.

\section{QUALITY OF LIFE}

The two patients with spinal cord injury whose spasms were controlled became more confident in using their wheelchairs and regained the ability to drive their cars; both reported improvements in their social life and one became employed. The other patients in whom the treatment was effective were much more comfortable sitting and one was enabled to sit as a result and go for trips with his family. Their carers found that nursing, dressing, toileting, and transferring became easier. 
Summary of patients treated, arranged in order of treatment from May 1986 to September 1991

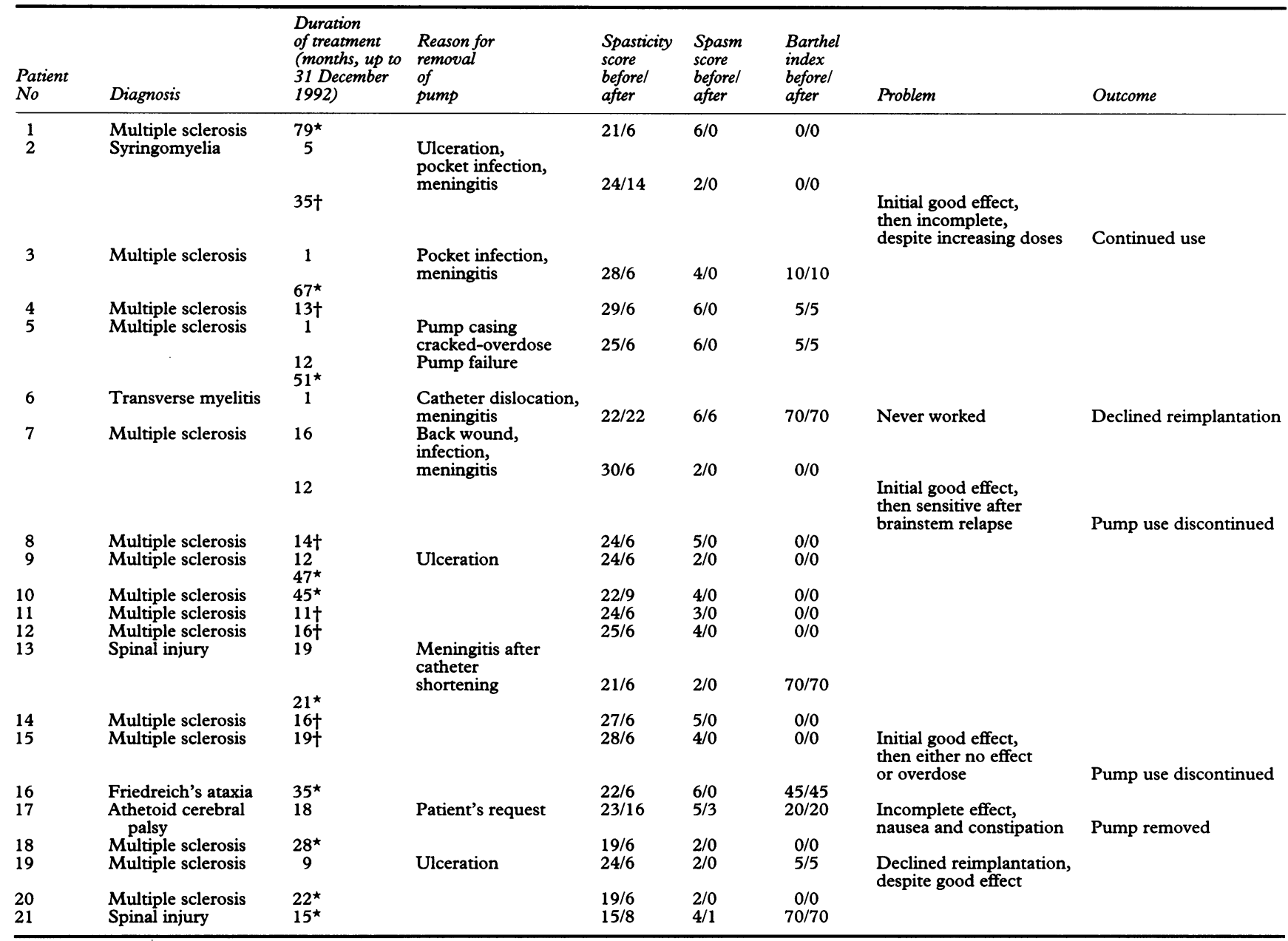

*Continues in use.

†Patient died.

Similar benefits were noted in the other four patients when the treatment was working.

DOSE REQUIRED AND DURATION OF EFFECT

The mean daily dose at the beginning of the trial was $223 \mu \mathrm{g}$ (range 50-400). The last effective dose was greater, with a mean of $485 \mu \mathrm{g}$ (range 100-1800).

\section{COMPLICATIONS}

During the study seven patients died from the effects of their disease without contribution from their intrathecal treatment. Significant complications which required the removal of the pump occurred in nine patients. Seven of these occurred in the first nine patients treated. Infection of the pump pocket occurred in two patients, both of whom developed secondary meningitis. One patient developed an infection of the back wound with secondary meningitis. Three patients developed erosions of the pump through the skin and one of these went on to develop a pocket infection and secondary meningitis. One episode of meningitis occurred for which there was no obvious wound infection. Culture of the pump fluid was sterile and the source remains uncertain. In each case coagulase-negative Staphylococcus aureus was the organism responsible and all patients responded rapidly to pump removal and intra- venous antibiotics. Two pump failures occurred, both in the same patient. The first of these was associated with a defect which allowed a baclofen overdose to occur due to a leak between the reservoir and the outlet tube; the second was due to failure of the buttons. Both pumps were replaced.

\section{BACLOFEN OVERDOSE}

Significant baclofen overdose with drowsiness, hypotension, and hypoventilation occurred in three patients, requiring a short period of support in the intensive care unit. Spontaneous recovery occurred in all patients within 24-48 hours with no new deficits.

\section{IN VITRO COMPATIBILITY}

Baclofen solution removed from the three pumps at seven weeks showed no significant change and lactam was not detected. At 18 weeks the mean baclofen concentration had decreased by $1 \%$ and lactam was detected at less than the $1 \%$ limit. Examination of the pumps by the manufacturers showed no adverse interaction.

IN VIVO STABILITY

With two exceptions the decrease in baclofen concentration was less than $2 \%$ (mean change $-1 \cdot 3 \%$; range $+2 \cdot 6 \%$ to $-10 \cdot 3 \%$ ). Lactam was not detected in five of the eight samples 
and was present at less than $1 \%$ in the remainder.

\section{Discussion}

Our results show that intrathecal baclofen is an effective treatment for severe spasticity in most patients treated. In patients with multiple sclerosis it has made a dramatic difference and the two patients who had severe spasms secondary to spinal cord injury have had complete relief of their spasms, with marked improvement in their lifestyle. In most patients there was also a significant improvement in the quality of life for patients and carers, indicating that spasticity was an important impairment.

Our studies suggest that baclofen is compatible with the Secor pump and that there is no adverse interaction between the pump and the baclofen. The baclofen solution seemed to be stable in vivo for the duration for which it is likely to remain within the pump.

The dose of baclofen which is required to control spasticity does vary between patients and, like other workers, we found that increased amounts were sometimes required with time, indicating what is probably a true pharmacological tolerance.

This success was achieved at the expense of significant complications. Most of our serious complications occurred in the first patients which we treated and, almost certainly, represent inexperience with what is a complicated technique. The implantation of the pumps, though superficially simple, provides many opportunities for things to go wrong. Such factors as the depth of the pocket and the sequence in which the operation is performed appear to be important. Some complications, particularly erosion due to accidental trauma to the pumps, as appeared to happen in patient 19 , are probably unavoidable. All but two of the patients who had to have pumps removed asked for a further pump to be implanted.

It should be noted that these patients were all severely disabled. None was ambulant and some were entirely dependent on their carers, as can be seen from their Barthel scores. The seven patients who died during the study did so from complications of their underlying neurological disease, and none of the deaths were related to either baclofen or its complications.

These results are comparable in terms of effectiveness with the other studies in which intrathecal baclofen has been given over an extended period. ${ }^{9-10}$ These have used more complicated pumps which are either externally programmable or continuous infusion. The comparability of the results and the low degree of dosage precision which is required with intrathecal baclofen for non-ambulant patients do not indicate that these more complicated pumps are of any advantage. The types of side effects encountered appear qualitatively similar in all studies with intrathecal baclofen.

It is difficult to say how intrathecal baclofen treatment compares with more conventional methods of treatment for severe spasticity, such as intrathecal phenol and commissural myelotomy. Our experience of these techniques has been less impressive than that reported elsewhere. ${ }^{11}$ Indeed, three of our patients had had intrathecal phenol treatment carried out unsuccessfully and one had had a commissural myelotomy, again unsuccessfully, before their pumps were implanted. We suggest that intrathecal baclofen is an effective treatment for severe spasticity in most patients and that a manually operated pump is probably as effective in delivering this drug to severely disabled patients as more sophisticated pumps.

We are grateful to the staff in the central pharmaceutical production unit and the pharmacy department, Royal Victoria Hospital for the preparation of the baclofen solutions and to Dr Frederick Feith for advice and support.

1 Penn RD, Kroin JS. Intrathecal baclofen alleviates spinal cord spasticity. Lancet 1984;i:1078.

2 Penn RD, Kroin JS. Continuous intrathecal baclofen for severe spasticity. Lancet 1985;ii:125-7.

3 Ashworth B. Preliminary trial of carisoprodol in multiple sclerosis. Practitioner 1964;192:540-2.

4 Lazorthes Y, Verdie JC, Bastide R, Lavados A, Descouens $D$. Spinal versus intraventricular chronic opiate administration with implantable drug delivery devices for cancer pain. Appl Neurophysiol 1985;48:234-41.

5 Borka $\mathrm{L}$. The formation, structure and analysis of the thermal decomposition product of baclofen. Acta Pharm Suecica 1979;16:345-8.

6 British Pharmacopoeia. Vol. 1. London: Pharmaceutical Press, 1988:54

7 United States Pharmacopeia. 21st revision. Suppl 5. Rockville: United States Pharmocopeia Convention, 1987:2343.

8 Harrison RM. Determination of 4-amino-3-(pchloropheny)-butyric acid (baclofen) in plasma by highperformance liquid chromatography. $\mathcal{F}$ Chromatogr 1985; performance

9 Penn RD. Intrathecal baclofen for spasticity of spinal origin: seven years of experience. $\mathcal{F}$ Neurosurg 1992;77: $236-40$.

10 Lazorthes Y, Sallerin-Caute B, Verdie J-C, Bastide R, Carillo J-P. Chronic intrathecal baclofen administration for control of severe spasticity. $\mathcal{F}$ Neurosurg 1990; 72:393-402.

11 Putty TK, Shapiro S. Efficacy of dorsal longitudinal myelotomy in treating spinal spasticity: a review of twenty cases. $\mathcal{f}$ Neurosurg 1991;75:397-401. 